(C2008 IEEE. Personal use of this material is permitted. However, permission to reprint/republish this material for advertising or promotional purposes or for creating new collective works for resale or redistribution to servers or lists, or to reuse any copyrighted component of this work in other works must be obtained from the IEEE 


\title{
On Sequential Frame Synchronization in AWGN Channels
}

\author{
Marco Chiani, Senior Member, IEEE, and Maria G. Martini, Member, IEEE
}

\begin{abstract}
We present a framework for the analysis of frame synchronization based on synchronization words (SWs), where the detection is based on the following sequential algorithm. The received samples are observed over a window of length equal to the SW; over this window, a metric (e.g., correlation) is computed; an SW is declared if the computed metric is greater than a proper threshold, otherwise the observation window is time-shifted one sample. We assume a Gaussian channel, antipodal signaling, equally distributed data symbols, and coherent detection, where soft values are provided to the frame synchronizer. We state the problem starting from the hypothesis testing theory, deriving the optimum metric [optimum likelihood ratio test (LRT)] according to the Neyman-Pearson lemma. When the data distribution is unknown, we design a simple and effective test based on the generalized LRT (GLRT). We also analyze the performance of the commonly used correlation metric, both with "hard" and "soft" values at the synchronizer input. We show that synchronization can be greatly improved by using the LRT and GLRT metrics instead of correlation and that, among correlation-based tests, sometimes hard correlation is better than soft correlation. The obtained closed-form expressions allow the derivation of the receiver operating characteristic (ROC) curves for the LRT and GLRT synchronizers, showing a remarkable gain with respect to synchronization based on correlation metric.
\end{abstract}

Index Terms-Barker sequences, detection, frame synchronization, generalized likelihood ratio test (GLRT), hypothesis testing, likelihood ratio test (LRT).

\section{INTRODUCTION}

$\mathbf{F}$ RAME synchronization is a critical issue in communication [1]-[4]. An example is video transmission where information is usually organized in frames, and decoding requires the knowledge of their exact boundaries.

This is the case, e.g., of the MPEG-4 video stream [5], [6], where synchronization markers (synchronization words (SWs) or start codes) are aperiodically embedded in the bitstream. The SW length and the detection strategy should be chosen, in this case, according to channel conditions and source characteristics.

In general, the quality degradation due to imperfect synchronization depends on the probability of missing the SW (missed detection) and on the probability of false start code detection

Paper approved by C. Tepedelenlioglu, the Editor for Transmission Systems of the IEEE Communications Society. Manuscript received March 21, 2005; revised August 3, 2005. This work was supported by the European Commission under Project FP6 IST-001812 "PHOENIX," and within the Network of Excellence NEWCOM. This paper was presented in part at the IEEE GLOBECOM 2004 Conference, Dallas, TX.

The authors are with the Consorzio Nazionale Interuniversitario per le Telecomunicazioni (CNIT), Dipartimento di Elettronica Informatica e Sistemistica, University of Bologna, 40136 Bologna, Italy (e-mail: mchiani@deis.unibo.it; mgmartini@deis.unibo.it).

Digital Object Identifier 10.1109/TCOMM.2005.863727 (false alarm). It is thus important to search for synchronization algorithms that minimize these probabilities and to provide their performance evaluation.

In the case of periodically embedded SWs, i.e., the case of fixed length frames of $N_{D}$ data symbols delimited by SWs of length $N$, frame synchronization can be performed through the search of the maximum of a metric in a window of $N_{f}=$ $N_{D}+N$ symbols. More precisely, for each of the possible $N_{D}+N$ positions of the $\mathrm{SW}$ in the observation window, a metric is evaluated over $N$ consecutive (modulo $N_{D}+N$ ) received symbols. The position of the SW is chosen as the one corresponding to the maximum evaluated metric. In the binary symmetric channel (BSC) case, the optimal metric is simply the correlation between the observed $N$-symbol sequence and the SW pattern [1]. In additive white Gaussian noise (AWGN) channels with soft outputs this is not true, as shown in [7], where the optimum metric for frame synchronization in the case of periodically embedded sync words is derived. However, regardless of its suboptimality, detection through correlation has become a common engineering practice even when soft values are available [3], [4].

Several studies have been performed considering periodically embedded sync words. The performance evaluation for binary symmetric channels has been studied in [1], where also synchronization sequences with good aperiodic autocorrelation properties have been identified. The generalization to polyphase sequences is studied, e.g., in [8] and [9].

In [3], a basic theory of frame synchronization is also presented, and considerations on the marker design are made. A frame sync acquisition algorithm, based on the comparison of the correlation metric with a threshold, is considered for the Gaussian channel with coherent demodulation. Also, in [3], following [7], a deferred-decision frame sync acquisition based on correlation is proposed in order to obtain a compromise between performance and simplicity. In [10], a union lower bound on synchronization probability for this acquisition rule on AWGN channels was determined. In [11], a performance evaluation through simulation of various metrics based on the search of the maximum in a fixed-length window has been presented. Depending on channel models and on applications, frame synchronization can be also aided by properly using information about erased bits or by joint frame synchronization and decoding [12], [13].

The aperiodically embedded case has been less considered in literature. The expected duration of a search for a fixed pattern in a semi-infinite stream of random data is addressed in [14], where its dependency on the structure of the pattern is also analyzed. The extension of the optimal frame sync approach [7] 
to nonbinary modulation schemes and to frames of known but not necessary constant lengths can be found in [15], where the search is based on the maximization of a proper metric over a fixed window; in the same work, numerical results describing the performance of the synchronizer are provided through simulation.

However, there is a lack of studies on optimal frame sync algorithms that are also valid for the general case of synchronization of frames of unknown variable lengths.

In this paper, the case of synchronization patterns aperiodically embedded in the bitstream is considered and afforded in the hypothesis testing framework [16], [17]. We assume that we do not have a priori information on the frame lengths. In this case, an acquisition algorithm based on a step-by-step comparison of a proper metric with a threshold is considered, and the detection metrics for AWGN channels based on the likelihood ratio test (LRT) and its generalized version are derived. An analytical performance evaluation of the LRT is given, assuming equiprobable data symbols. In order to benchmark the obtained results, the analysis of frame synchronization through the correlation metric is also reported, both for a BSC and for an AWGN channel model, and represented in terms of receiver operating characteristic (ROC) curves. Note that the derived synchronizer may be also used, as a particular case, for fixed-length frames.

It will be shown that the new metrics, derived according to LRT and generalized LRT (GLRT), provide large gains with respect to those based on correlation, both in the "soft" and "hard" versions. ${ }^{1}$

The paper is organized as follows. In Section II, the frame synchronization problem analyzed is described in detail. In Section III, the LRT and its generalized forms are derived. The analytical performance evaluation of the tests under investigation is provided in Section IV. Section V describes the performance of the correlation-based tests in an AWGN channel. Numerical results are reported in Section VI, where simulation results are also provided to validate the analysis. Finally, conclusions are drawn in Section VII.

\section{Problem Statement}

We first consider binary antipodal signaling, so that the $i$ th transmitted bit $b_{i} \in\{0,1\}$ gives rise, after binary antipodal modulation, transmission through the AWGN channel, matched filter reception, and perfect sampling, to a real sample $r_{i}=$ $(-1)^{b_{i}}+n_{i}$, where $n_{i}$ are independent identically distributed (i.i.d.) real Gaussian random variables (r.v.s), with zero mean and variance $\sigma^{2}$. This model is also valid for binary phaseshift keying (BPSK) systems, for which the signal-to-noise ratio (SNR) is $E_{s} / N_{0}=1 /\left(2 \sigma^{2}\right)$, where $E_{s}$ is the energy per symbol and $N_{0}$ is the one-sided thermal noise power spectral density.

We assume that an $N$-binary-symbols sync word $\left(c_{1}, \ldots, c_{N}\right)$ is aperiodically inserted in the data stream, composed of symbols $d_{i} \in\{-1,+1\}$ that are i.i.d. r.v.s with equiprobable -1 and +1 (see Fig. 1). Each SW symbol $c_{i}$ is either -1 or +1 .

\footnotetext{
${ }^{1}$ We refer to soft values as the real valued samples at the AWGN channel output and to hard values as their binary quantized version.
}

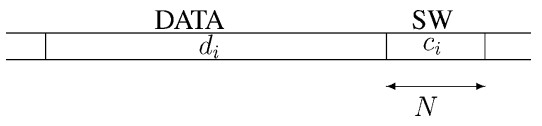

Fig. 1. Frame structure.

The acquisition algorithm we consider is as follows: starting from a position $k$, the synchronizer observes a vector of $N$ subsequent samples. Based on a suitable metric evaluated from this vector, it decides if the $\mathrm{SW}$ is in position $k$; if not, it moves to position $k+1$, repeating the steps until the sync word is detected.

In this paper, we afford the problem of deciding at each position $k$ of the bitstream whether a sync word is present or not. The relation between this problem and other performance indicators such as, e.g., the probability of correct acquisition in one pass, is addressed in [3] and [14].

We assume in the following that the statistical properties of the metric do not depend on the position in the bitstream. We thus avoid considering the effect of the aperiodic autocorrelation around SWs. We have evaluated in fact that, if the SW is properly chosen as a sequence with optimized aperiodic autocorrelation property (e.g., Barker sequences [1] or those in [3], [18], and [19]), then its symbols should mimic random data, with, moreover, the additional property that some configurations can be avoided.

We consider thus, without losing generality, $k=1$, and we neglect in the design and analytical performance evaluation of the frame synchronizer the case of "mixed data," i.e., when both data and SW symbols are present in the metric evaluation window. However, some results will be given in Section VI for the case of "mixed data" to support this approximation. In particular, we will show that, if the SW is properly designed, the case of purely random data represents generally a worst case in terms of probability of false sync word detection respect to the "mixed data" case.

We study the problem through the statistical theory of hypothesis testing. After observing $N$ subsequent samples, the synchronizer must choose between two possible hypotheses

$$
\begin{aligned}
& \mathcal{H}_{0}: r_{i}=d_{i}+n_{i}, \quad i=1, \ldots, N \\
& \mathcal{H}_{1}: r_{i}=c_{i}+n_{i}, \quad i=1, \ldots, N
\end{aligned}
$$

where the first one represents the case where there is no SW, and the second corresponds to the case where the SW is present. Decisions are indicated by $\mathcal{D}_{0}$ and $\mathcal{D}_{1}$, corresponding to hypotheses $\mathcal{H}_{0}$ and $\mathcal{H}_{1}$, respectively.

The probability of emulation $P_{\mathrm{EM}}$ or false start code detection $^{2}$ of choosing hypothesis $\mathcal{H}_{1}$ when $\mathcal{H}_{0}$ is true is

$$
P_{\text {EM }}=\operatorname{Pr}\left\{\mathcal{D}_{1} \mid \mathcal{H}_{0}\right\}
$$

The probability of missed detection $P_{\mathrm{MD}}$ of choosing $\mathcal{H}_{0}$ when $\mathcal{H}_{1}$ is true is

$$
P_{\mathrm{MD}}=\operatorname{Pr}\left\{\mathcal{D}_{0} \mid \mathcal{H}_{1}\right\}
$$

${ }^{2}$ Here, the false start code detection is due to the case where random data plus noise is interpreted as an SW. This can occur either in the case where data symbols are coincident with the SW pattern or, due to noise, even if data symbols are different from the SW pattern. 
and the probability of correct detection is

$$
P_{\mathrm{D}}=1-P_{\mathrm{MD}}
$$

\section{Sequential Frame Synchronization Tests}

\section{A. Derivation of the LRT}

Assuming AWGN and availability of soft values, the LRT [17] is considered for the derivation of the optimal detection metric. In the following, we use capital letters to indicate random variables and bold for vectors.

By indicating with $\mathbf{R}=\left(R_{1}, \ldots, R_{N}\right)$ the r.v. corresponding to the vector $\mathbf{r}=\left(r_{1}, \ldots, r_{N}\right)$ of received samples, the LRT is

$$
\Lambda^{\prime}(\mathbf{r})=\frac{f_{\mathbf{R} \mid \mathcal{H}_{0}}\left(\mathbf{r} \mid \mathcal{H}_{0}\right)}{f_{\mathbf{R} \mid \mathcal{H}_{1}}\left(\mathbf{r} \mid \mathcal{H}_{1}\right)} \underset{\mathcal{D}_{1}}{\stackrel{\mathcal{D}_{0}}{\gtrless}} \lambda^{\prime}
$$

where $f_{\mathbf{R} \mid \mathcal{H}_{l}}\left(\mathbf{r} \mid \mathcal{H}_{l}\right)$ is the probability density function (pdf) of $\mathbf{R}$ under hypothesis $\mathcal{H}_{l}, l \in\{0,1\}$, and $\lambda^{\prime}$ is the selected threshold. Thus, according to the test, $\Lambda^{\prime}(\mathbf{r})<\lambda^{\prime}$ corresponds to the decision $\mathcal{D}_{1}$, i.e., we decide we are in presence of an $\mathrm{SW}$; otherwise, the decision is $\mathcal{D}_{0}$. Since the channel is memoryless, we have also

$$
f_{\mathbf{R} \mid \mathcal{H}_{l}}\left(\mathbf{r} \mid \mathcal{H}_{l}\right)=\prod_{i=1}^{N} f_{R_{i} \mid \mathcal{H}_{l}}\left(r_{i} \mid \mathcal{H}_{l}\right)
$$

Let us note that, given $\mathcal{H}_{1}$, the $R_{i}$ are Gaussian-distributed r.v.s with mean $c_{i}$ and variance $\sigma^{2}$, i.e.,

$$
f_{R_{i} \mid \mathcal{H}_{1}}\left(r_{i} \mid \mathcal{H}_{1}\right)=\frac{1}{\sqrt{2 \pi} \sigma} e^{-\left(r_{i}-c_{i}\right)^{2} / 2 \sigma^{2}}
$$

Now, we observe that, conditioned on $\mathcal{H}_{0}$ and $d_{i}, R_{i}$ is a Gaussian r.v. with variance $\sigma^{2}$ and mean $d_{i}$, i.e.,

$$
f_{R_{i} \mid \mathcal{H}_{0}, d_{i}}\left(r_{i} \mid \mathcal{H}_{0}, d_{i}\right)=\frac{1}{\sqrt{2 \pi} \sigma} e^{-\left(r_{i}-d_{i}\right)^{2} / 2 \sigma^{2}} .
$$

Since, under hypothesis $\mathcal{H}_{0}$, symbols $d_{i}$ take values +1 and -1 with equal probability, we get

$$
\begin{aligned}
f_{R_{i} \mid \mathcal{H}_{0}}\left(r_{i} \mid \mathcal{H}_{0}\right)= & \frac{1}{2} f_{R_{i} \mid \mathcal{H}_{0}, d_{i}}\left(r_{i} \mid \mathcal{H}_{0}, d_{i}=1\right) \\
& +\frac{1}{2} f_{R_{i} \mid \mathcal{H}_{0}, d_{i}}\left(r_{i} \mid \mathcal{H}_{0}, d_{i}=-1\right) .
\end{aligned}
$$

Therefore, using (7)-(9), the metric given in (5) becomes

$$
\begin{aligned}
\Lambda^{\prime}(\mathbf{r}) & =\frac{1}{2^{N}} \prod_{i=1}^{N} \frac{e^{-\left(r_{i}-1\right)^{2} / 2 \sigma^{2}}+e^{-\left(r_{i}+1\right)^{2} / 2 \sigma^{2}}}{e^{-\left(r_{i}-c_{i}\right)^{2} / 2 \sigma^{2}}} \\
& =\frac{1}{2^{N}} \prod_{i=1}^{N}\left(1+e^{-2 r_{i} c_{i} / \sigma^{2}}\right)
\end{aligned}
$$

that, neglecting the inessential factor $1 / 2^{N}$, can be equivalently written as

$$
\Lambda^{\prime \prime}(\mathbf{r})=\prod_{i=1}^{N}\left(1+e^{-2 r_{i} c_{i} / \sigma^{2}}\right)
$$

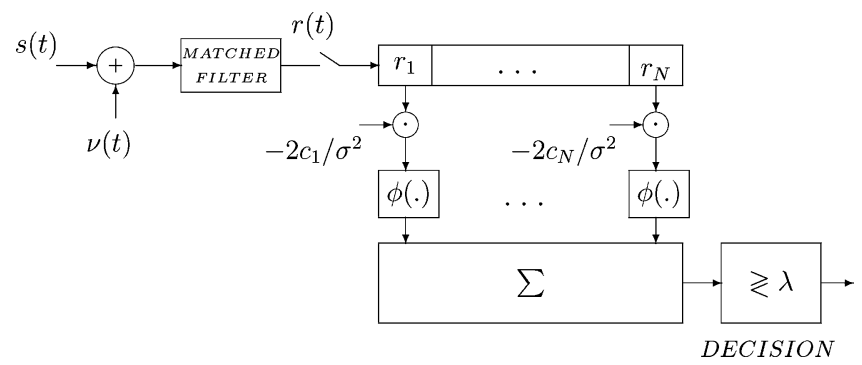

Fig. 2. Optimal sequential synchronizer scheme, $\phi(x)=\ln \left(1+e^{x}\right)$.

Then, by applying the logarithm we obtain the log-likelihood ratio test (LLRT) as

$$
\Lambda=\Lambda(\mathbf{r})=\sum_{i=1}^{N} \ln \left(1+e^{-2 r_{i} c_{i} / \sigma^{2}}\right) \underset{\mathcal{D}_{1}}{\stackrel{\mathcal{D}_{0}}{\gtrless} \lambda .}
$$

We thus decide $\mathcal{D}_{1}$ (the start code is present) if $\Lambda(\mathbf{r})<\lambda$ and $\mathcal{D}_{0}$ otherwise. The threshold $\lambda$ is chosen according to the Neyman-Pearson criterion, i.e., by fixing the maximum tolerable probability of false alarm (emulation). We should note that the obtained metric depends on channel conditions through $\sigma^{2}$, and thus the synchronizer requires the instantaneous knowledge of the signal-to-noise ratio (SNR) in order to perform detection. Furthermore, the evaluation of a nonlinear function is required. The LRT-based synchronizer is depicted in Fig. 2, where $\phi(x)=\ln \left(1+e^{x}\right)$.

Note that the previous derivation of the test can be easily extended to cover M-ary modulation formats and nonequiprobable data. To this aim, it is sufficient to consider (1) with (in general complex) data symbols $d_{i} \in\left\{d_{(1)}, d_{(2)}, \ldots, d_{(M)}\right\}$, SW symbols $c_{i} \in\left\{d_{(1)}, d_{(2)}, \ldots, d_{(M)}\right\}$, and (in general complex) noise samples $n_{i}$ with i.i.d. real and imaginary components. The LRT is then obtained as above by observing that $R_{i} \mid \mathcal{H}_{1}$ is complex Gaussian with mean value $c_{i}$ and by replacing (9) with

$$
f_{R_{i} \mid \mathcal{H}_{0}}\left(r_{i} \mid \mathcal{H}_{0}\right)=\sum_{k=1}^{M} \operatorname{Pr}\left\{d_{i}=d_{(k)}\right\} f_{R_{i} \mid \mathcal{H}_{0}, d_{i}}\left(r_{i} \mid \mathcal{H}_{0}, d_{i}=d_{(k)}\right)
$$

where $R_{i} \mid \mathcal{H}_{0}, d_{i}=d_{(k)}$ is a complex Gaussian r.v. with mean value $d_{(k)}$.

In the following, for the sake of simplicity and conciseness, we will focus the analysis on the case of binary equiprobable symbols.

\section{B. Derivation of the GLRT}

In order to benchmark the performance of the previously derived metric, we here present another possible test that will be shown to have some advantages in terms of implementation complexity.

In particular, in [20], we studied frame synchronization for those situations where we cannot make any assumption about the probability distribution of data symbols $d_{i}$. In this case, we may resort to the generalized LRT (GLRT) [17] for the design of the detection strategy, since designing the LRT requires knowledge of the data distribution. The GLRT approach leads, in principle, to a two-step procedure. First, we estimate the unknown data vector $\mathbf{d}=\left(d_{1}, \ldots, d_{N}\right)$ under hypothesis $\mathcal{H}_{0}$. Then, we 
use the estimate $\widehat{\mathbf{d}}=\left(\widehat{d}_{1}, \ldots, \widehat{d}_{N}\right)$ as if it were the correct value of the transmitted data. The GLRT is

$$
\Lambda_{g}^{\prime}(\mathbf{r})=\frac{f_{\mathbf{R} \mid \mathcal{H}_{1}}\left(\mathbf{r} \mid \mathcal{H}_{\mathbf{1}}\right)}{f_{\mathbf{R} \mid \mathcal{H}_{0}, \mathbf{d}}\left(\mathbf{r} \mid \mathcal{H}_{0}, \widehat{\mathbf{d}}\right)} \underset{\mathcal{D}_{0}}{\stackrel{\mathcal{D}_{1}}{\gtrless}} \lambda^{\prime}
$$

where $\lambda^{\prime}$ is the selected threshold. In our case, we use the maximum-likelihood estimate for each of the $d_{i}$, which is clearly $\widehat{d}_{i}=\operatorname{sign}\left(r_{i}\right)$ for $i=1, \ldots, N$.

Starting from (14), the GLRT becomes [20]

$$
\Lambda_{g}=\Lambda_{g}(\mathbf{r})=\sum_{i=1}^{N}\left(\left|r_{i}\right|-r_{i} c_{i}\right) \stackrel{\mathcal{D}_{0}}{\underset{\mathcal{D}_{1}}{\gtrless}} \lambda
$$

where $\lambda \propto \ln \left(\lambda^{\prime}\right)$. Note that, since $\left|c_{i}\right|=1$, the quantity to be evaluated in test (15) can be interpreted as twice the sum of the absolute value of the samples $r_{i}$ whose signs differ from that of the SW symbols $c_{i}$. In other words, the GLRT-based synchronizer can just discard those samples whose signs are equal to that of the SW and compare the sum of the remaining (absolute values) with a threshold.

We observe that this metric does not depend on the SNR, and it may thus be used also when information on the channel conditions is not available.

Hence, from an implementation point of view, the synchronizer based on the GLRT (15) is simpler than that based on the optimum LRT (12). We will see later that, despite its simplicity, the GLRT performs very similarly to LRT except for very low SNRs. Indeed, since the function $\ln \left(1+e^{x}\right)$ may be approximated by 0 for $x \ll 0$ and by $x$ for $x \gg 0$, it is simple to verify that the LRT in (12) tends to the GLRT for increasing SNRs. Moreover, the GLRT does not need any assumption about data distribution (i.e., $\operatorname{Pr}\left\{d_{i}=+1\right\}$ ), whereas, in order to design the optimal LRT, this distribution is required at the synchronizer [see (13)]. From this point of view, it is worthwhile to remark that the test in (12), that has been designed for equiprobable data, loses its optimality when applied to unbalanced data symbols.

\section{Performance Evaluation of SEQUENTIAL TESTS ON AWGN}

In this section, we provide the performance evaluation of the presented tests in terms of probability of false start code detection, or emulation, and of probability of missed detection.

First, let us observe that the probability of emulation $P_{\mathrm{EM}}$ can be evaluated as

$$
P_{\mathrm{EM}}=\operatorname{Pr}\left\{\Lambda_{x}<\lambda \mid \mathcal{H}_{0}\right\}=F_{\Lambda_{x} \mid \mathcal{H}_{0}}\left(\lambda \mid \mathcal{H}_{0}\right)
$$

where $\Lambda_{x} \in\left\{\Lambda, \Lambda_{g}\right\}$ represents the considered metric, $\lambda$ is the selected threshold in (12) or (15), and $F_{\Lambda_{x} \mid \mathcal{H}_{l}}($.$) is the cumu-$ lative distribution function (cdf) of $\Lambda_{x}$ under hypothesis $\mathcal{H}_{l}$, $l \in\{0,1\}$. Similarly, the probability of missed detection $P_{\mathrm{MD}}$ is given by

$$
P_{\mathrm{MD}}=\operatorname{Pr}\left\{\Lambda_{x}>\lambda \mid \mathcal{H}_{1}\right\}=1-F_{\Lambda_{x} \mid \mathcal{H}_{1}}\left(\lambda \mid \mathcal{H}_{1}\right) .
$$

Thus, the performance analysis of the synchronizer requires the evaluation of the cdfs of the involved metric.

\section{A. Performance Analysis of LRT}

In order to evaluate the performance of the optimal test, we need to evaluate the statistical distribution of the r.v. $\Lambda(\mathbf{R})$ as defined in (12), for the two hypotheses $\mathcal{H}_{0}, \mathcal{H}_{1}$.

To this aim, we first study the distribution of the auxiliary r.v.s

$$
W_{i}=1+\exp \left\{-\frac{2 R_{i} c_{i}}{\sigma^{2}}\right\}>1
$$

and

$$
Z_{i}=\ln \left(W_{i}\right)
$$

so that the statistical analysis of the LLR in (12) is derived by studying the r.v.

$$
\Lambda(\mathbf{R})=\sum_{i=1}^{N} \ln W_{i}=\sum_{i=1}^{N} Z_{i} .
$$

Under hypothesis $\mathcal{H}_{l}, l \in\{0,1\}$, the cdf of $W_{i}$ is

$$
\begin{aligned}
F_{W_{i} \mid \mathcal{H}_{l}}\left(w \mid \mathcal{H}_{l}\right) & =\operatorname{Pr}\left\{W_{i} \leq w \mid \mathcal{H}_{l}\right\} \\
& =\operatorname{Pr}\left\{1+e^{-2 R_{i} c_{i} / \sigma^{2}} \leq w \mid \mathcal{H}_{l}\right\} .
\end{aligned}
$$

We then have

$$
\begin{aligned}
F_{W_{i} \mid \mathcal{H}_{l}}\left(w \mid \mathcal{H}_{l}\right) & =\operatorname{Pr}\left\{R_{i} c_{i} \geq-\sigma^{2} \ln \sqrt{w-1} \mid \mathcal{H}_{l}\right\} \\
& =1-F_{\Psi_{i} \mid \mathcal{H}_{l}}\left(-\sigma^{2} \ln \sqrt{w-1} \mid \mathcal{H}_{l}\right)
\end{aligned}
$$

where $F_{\Psi_{i} \mid \mathcal{H}_{l}}($.$) is the cdf of \Psi_{i}=R_{i} c_{i}$ given $\mathcal{H}_{l}$.

Using the basic theory for the transformation of r.v.s, we also have from (19) the relation $F_{Z_{i} \mid \mathcal{H}_{l}}\left(z \mid \mathcal{H}_{l}\right)=F_{W_{i} \mid \mathcal{H}_{l}}\left(e^{z} \mid \mathcal{H}_{l}\right)$, with $z \geq 0$.

1) LLR Statistical Distribution Under $\mathcal{H}_{0}$ : Since, under hypothesis $\mathcal{H}_{0}$, the r.v. $R_{i}$ is symmetric, then $\Psi_{i}=R_{i} c_{i}$ has the same distribution, i.e., both have the pdf (9). By applying (21), we then have

$$
\begin{aligned}
F_{W_{i} \mid \mathcal{H}_{0}}\left(w \mid \mathcal{H}_{0}\right)= & \frac{1}{4} \operatorname{erfc}\left(\frac{-1-\sigma^{2} \ln \sqrt{w-1}}{\sqrt{2} \sigma}\right) \\
& +\frac{1}{4} \operatorname{erfc}\left(\frac{1-\sigma^{2} \ln \sqrt{w-1}}{\sqrt{2} \sigma}\right)
\end{aligned}
$$

where

$$
\operatorname{erfc}(x) \triangleq \frac{2}{\sqrt{\pi}} \int_{x}^{\infty} e^{-t^{2}} d t
$$

is the complementary Gaussian error function. Note that $\operatorname{erfc}(-x)=2-\operatorname{erfc}(x)$ and that, for $x>0$, the function can be also efficiently evaluated as $\operatorname{erfc}(x)=(2 / \pi)$ $\int_{0}^{\pi / 2} \exp \left(-x^{2} / \sin ^{2} \theta\right) d \theta$, thus providing the simple exponential approximation $\operatorname{erfc}(x) \simeq(1 / 6) e^{-x^{2}}+(1 / 2) e^{-(4 / 3) x^{2}}$ [21].

For the r.v. $Z_{i}$, we have

$$
\begin{aligned}
F_{Z_{i} \mid \mathcal{H}_{0}}\left(z \mid \mathcal{H}_{0}\right)= & \frac{1}{4} \operatorname{erfc}\left(\frac{-1-\sigma^{2} \ln \sqrt{e^{z}-1}}{\sqrt{2} \sigma}\right) \\
& +\frac{1}{4} \operatorname{erfc}\left(\frac{1-\sigma^{2} \ln \sqrt{e^{z}-1}}{\sqrt{2} \sigma}\right) .
\end{aligned}
$$


Taking the derivative with respect to $z$, we obtain the pdf

$$
\begin{aligned}
f_{Z_{i} \mid \mathcal{H}_{0}}\left(z \mid \mathcal{H}_{0}\right)= & \frac{\sigma}{4 \sqrt{2 \pi}} \frac{e^{z}}{e^{z}-1} \\
& \cdot\left[e^{-\left(1 / 2 \sigma^{2}\right)\left(1+\sigma^{2} \ln \sqrt{e^{z}-1}\right)^{2}}\right. \\
& \left.+e^{-\left(1 / 2 \sigma^{2}\right)\left(1-\sigma^{2} \ln \sqrt{e^{z}-1}\right)^{2}}\right] .
\end{aligned}
$$

We now recall that the characteristic function (ch.f.) of the sum of independent random variables is the product of the ch.f.s of the single terms. Since we are interested in the distribution of $\Lambda(\mathbf{R})$ that is the sum of $N$ independent r.v.s each with pdf (25), it is convenient to evaluate the ch.f. of the r.v. $Z_{i}$, here defined as

$$
\Phi_{Z_{i} \mid \mathcal{H}_{0}}(\nu) \triangleq \mathbb{E}\left[e^{j 2 \pi \nu Z_{i}}\right]=\int_{-\infty}^{\infty} f_{Z_{i} \mid \mathcal{H}_{0}}(z) e^{j 2 \pi \nu z} d z
$$

where $j=\sqrt{-1}$ is the imaginary unit.

Starting from the ch.f., both the pdf and cdf can be easily evaluated from (25) by using standard fast Fourier transform (FFT) techniques. For example, we have

$$
f_{\Lambda \mid \mathcal{H}_{0}}\left(\lambda \mid \mathcal{H}_{0}\right)=\mathcal{F}\left\{\left[\Phi_{Z_{i} \mid \mathcal{H}_{0}}(\nu)\right]^{N}\right\}
$$

where we indicated with $\mathcal{F}\{$.$\} the Fourier transform. Similarly,$ the cdf can be obtained as

$$
F_{\Lambda \mid \mathcal{H}_{0}}\left(\lambda \mid \mathcal{H}_{0}\right)=\int_{-\infty}^{\infty}\left[\Phi_{Z_{i} \mid \mathcal{H}_{0}}(\nu)\right]^{N}\left[\frac{1-e^{-j 2 \pi \lambda \nu}}{j 2 \pi \nu}\right] d \nu .
$$

This is then used in (16) to get the probability of false alarm.

2) LLR Statistical Distribution Under $\mathcal{H}_{1}$ : We consider now the case in which the SW is present. In this situation, an error is made if the SW is not detected. We here derive the probability $P_{\mathrm{MD}}=\operatorname{Pr}\left\{\mathcal{D}_{0} \mid \mathcal{H}_{1}\right\}$.

From (1), it results that $\Psi_{i}\left|\mathcal{H}_{1}=R_{i} c_{i}\right| \mathcal{H}_{1}$ is a Gaussian r.v. with mean $c_{i}^{2}$ and variance $\sigma^{2}$. Consequently, with the positions made above, from (21), we get

$$
F_{W_{i} \mid \mathcal{H}_{1}}\left(w \mid \mathcal{H}_{1}\right)=1-\frac{1}{2} \operatorname{erfc}\left(\frac{c_{i}^{2}+\sigma^{2} \ln \sqrt{w-1}}{\sqrt{2} \sigma}\right) .
$$

By substituting, since $c_{i}^{2}=1$, we have

$$
F_{Z_{i} \mid \mathcal{H}_{1}}\left(z \mid \mathcal{H}_{1}\right)=1-\frac{1}{2} \operatorname{erfc}\left(\frac{1+\sigma^{2} \ln \sqrt{e^{z}-1}}{\sqrt{2} \sigma}\right) .
$$

Taking the derivative with respect to $z$, we obtain

$$
f_{Z_{i} \mid \mathcal{H}_{1}}\left(z \mid \mathcal{H}_{1}\right)=\frac{\sigma}{2 \sqrt{2 \pi}} \frac{e^{z}}{e^{z}-1} e^{-\left(1 / 2 \sigma^{2}\right)\left(1+\sigma^{2} \ln \sqrt{e^{z}-1}\right)^{2}}
$$

and, therefore, indicating by $\Phi_{Z_{i} \mid \mathcal{H}_{1}}(\nu)$ the corresponding characteristic function, we get

$$
f_{\Lambda \mid \mathcal{H}_{1}}\left(\lambda \mid \mathcal{H}_{1}\right)=\mathcal{F}\left\{\left[\Phi_{Z_{i} \mid \mathcal{H}_{1}}(\nu)\right]^{N}\right\} .
$$

Again, this function and the cdf can be easily evaluated from (31) by using standard FFT techniques.

\section{B. Performance Analysis of GLRT}

We provide in this subsection a performance analysis of the GLRT synchronizer for equiprobable data symbols. We first ob- serve that, since $\left|c_{i}\right|=1$, we can equivalently write the metric in (15) as

$$
\Lambda_{g}=\sum_{i=1}^{N}\left|r_{i} c_{i}\right|-r_{i} c_{i}=\sum_{i=1}^{N} v_{i}
$$

where $v_{i}=\left|\psi_{i}\right|-\psi_{i}$ with $\psi_{i}=r_{i} c_{i}$. Hence

$$
v_{i}= \begin{cases}0, & \text { if } \psi_{i} \geq 0 \\ -2 \psi_{i}, & \text { otherwise }\end{cases}
$$

From (34), indicating with $f_{\Psi_{i} \mid \mathcal{H}_{l}}($.$) the pdf of \Psi_{i}$ under hypothesis $\mathcal{H}_{l}, l=0,1$ and by using the rules for transformation of random variables, the pdf of $V_{i}$ is

$$
f_{V_{i} \mid \mathcal{H}_{l}}(v)=\frac{1}{2} f_{\Psi_{i} \mid \mathcal{H}_{l}}\left(-\frac{v}{2}\right) u(v)+\operatorname{Pr}\left\{\Psi_{i} \geq 0 \mid \mathcal{H}_{l}\right\} \delta(v)
$$

where $u(\cdot)$ is the unitary step function and $\delta(\cdot)$ is the Dirac delta function.

By using (35), we obtain

$$
\begin{aligned}
\Phi_{V_{i} \mid \mathcal{H}_{l}}(\nu) & =\mathbb{E}\left[e^{j 2 \pi \nu V_{i}}\right] \\
& =\operatorname{Pr}\left\{\Psi_{i} \geq 0 \mid \mathcal{H}_{l}\right\}+\frac{1}{2} \int_{0}^{\infty} f_{\Psi_{i} \mid \mathcal{H}_{l}}\left(-\frac{v}{2}\right) e^{j 2 \pi \nu v} d v .
\end{aligned}
$$

Then, the ch.f. of $\Lambda_{g}(\mathbf{R})$ is simply

$$
\Phi_{\Lambda_{g} \mid \mathcal{H}_{l}}(\nu)=\prod_{i=1}^{N} \Phi_{V_{i} \mid \mathcal{H}_{l}}(\nu) .
$$

We now specialize (36) to the two hypotheses $\mathcal{H}_{0}$ and $\mathcal{H}_{1}$.

1) Case $\mathcal{H}_{0}$ : We first consider the case where the $S W$ is not present and the metric is thus applied to random data. The pdf of $R_{i} \mid \mathcal{H}_{0}$ is given in (9). This is clearly a symmetric r.v.; therefore, as before, we observe that $\Psi_{i}=R_{i} c_{i}$ has the same distribution, i.e.,

$$
f_{\Psi_{i} \mid \mathcal{H}_{0}}(\psi)=\frac{1}{2} \frac{1}{\sqrt{2 \pi} \sigma} e^{-(\psi-1)^{2} / 2 \sigma^{2}}+\frac{1}{2} \frac{1}{\sqrt{2 \pi} \sigma} e^{-(\psi+1)^{2} / 2 \sigma^{2}}
$$

and $\operatorname{Pr}\left\{\Psi_{i} \geq 0 \mid \mathcal{H}_{0}\right\}=1 / 2$. Substituting in (36) and by using the identity [22]

$$
\int_{0}^{\infty} e^{-\left(a t^{2}+2 b t+c\right)} d t=\frac{1}{2} \sqrt{\frac{\pi}{a}} e^{\left(b^{2}-a c\right) / a} \operatorname{erfc} \frac{b}{\sqrt{a}}
$$

with the real part of $a$ greater than 0 , we can express the ch.f. in closed form as

$$
\begin{aligned}
\Phi_{V_{i} \mid \mathcal{H}_{0}}(\nu)= & \frac{1}{4} e^{-4 \pi \nu\left(j+2 \pi \nu \sigma^{2}\right)} \\
& \cdot\left[\operatorname{erfc}\left(\frac{1-j 4 \pi \nu \sigma^{2}}{\sqrt{2} \sigma}\right)\right. \\
& \left.\quad+e^{j 8 \pi \nu} \operatorname{erfc}\left(\frac{-1-j 4 \pi \nu \sigma^{2}}{\sqrt{2} \sigma}\right)\right]+\frac{1}{2} .
\end{aligned}
$$

Finally, we may observe that, for the considered case of equiprobable data symbols, the particular SW pattern does not play any role. On the other side, SW patterns must be properly designed for the mixed data case, as previously discussed (see also Section VI). 
2) Case $\mathcal{H}_{1}:$ We here consider the case where the $\mathrm{SW}$ is present. Under this hypothesis, the generic $R_{i}$ is Gaussian distributed, with mean $c_{i}$ and variance $\sigma^{2}$. Thus, whatever $c_{i}$ is, the r.v. $\Psi_{i}=R_{i} c_{i}$ has a Gaussian pdf with mean value $c_{i}^{2}=1$, i.e.,

$$
f_{\Psi_{i} \mid \mathcal{H}_{1}}(\psi)=\frac{1}{\sqrt{2 \pi} \sigma} e^{-(\psi-1)^{2} / 2 \sigma^{2}}
$$

and consequently

$$
\operatorname{Pr}\left\{\Psi_{i} \geq 0 \mid \mathcal{H}_{1}\right\}=\frac{1}{2} \operatorname{erfc}\left(-\frac{1}{\sqrt{2} \sigma}\right) .
$$

Hence, from (36) and (41), we obtain

$$
\begin{aligned}
\Phi_{V_{i} \mid \mathcal{H}_{1}}(\nu)= & \frac{1}{2} \operatorname{erfc}\left(-\frac{1}{\sqrt{2} \sigma}\right) \\
& +\frac{1}{2} e^{-4 \pi \nu\left(j+2 \pi \nu \sigma^{2}\right)} \operatorname{erfc}\left(\frac{1-j 4 \pi \nu \sigma^{2}}{\sqrt{2} \sigma}\right) .
\end{aligned}
$$

In conclusion, (40) and (42) substituted into (37) give in closed form the ch.f. of the decision variable.

\section{Performance Evaluation of the Correlation Metric}

\section{A. Synchronizing With Hard Decisions (BSC Channel)}

In this section, for the purpose of comparison, the probabilities of missed detection and of emulation will be briefly reviewed by assuming that only hard decisions (i.e., $\operatorname{sign}\left(r_{i}\right)$ ) about the received samples $r_{i}$ are available, i.e., with a memoryless BSC channel assumption ${ }^{3}$ [1], [3].

According to the hypothesis testing theory, for BSC, the optimal test consists in the comparison of the correlation $\Gamma(\mathbf{r})=$ $\sum_{i=1}^{N} c_{i} \operatorname{sign}\left(r_{i}\right)$ with a prefixed threshold $\lambda$

$$
\Gamma(\mathbf{r})=\sum_{i=1}^{N} c_{i} \operatorname{sign}\left(r_{i}\right) \underset{\mathcal{D}_{0}}{\stackrel{\mathcal{D}_{1}}{\gtrless}} \lambda .
$$

In this test, we choose to decide for $\mathcal{D}_{1}$ in the case of equality.

We remark that $\Gamma(\mathbf{R})$ is a discrete r.v. and, consequently, with the above defined test, only certain values of $P_{\mathrm{EM}}, P_{\mathrm{MD}}$ are possible. A continuous variation could be obtained by introducing a randomized decision rule [17].

The probability of emulation is the probability that the correlation with a random sequence in the corrupted data bitstream exceeds the prefixed threshold $\lambda$

$$
P_{\mathrm{EM}}=\operatorname{Pr}\left\{\Gamma(\mathbf{R}) \geq \lambda \mid \mathcal{H}_{0}\right\} .
$$

The pdf of the correlation in (43) under $\mathcal{H}_{0}$ is

$$
f_{\Gamma}(\gamma)=\left(\frac{1}{2}\right)^{N} \sum_{i=0}^{N}\left(\begin{array}{c}
N \\
i
\end{array}\right) \delta(\gamma-N+2 i)
$$

where $(1 / 2)^{N}$ is the probability of the generic $n$-tuple, $\left(\begin{array}{c}N \\ i\end{array}\right)$ gives the number of sequences with $i$ symbols coincident with that of the SW, and $\delta($.$) is the Dirac's function.$

\footnotetext{
${ }^{3}$ As usual, we assume a BSC error probability $p<1 / 2$.
}

Summing from the threshold value $\lambda$ to $N$, we obtain the probability that the bitstream can emulate a start code as

$$
P_{\mathrm{EM}}=\left(\frac{1}{2}\right)^{N} \sum_{k=0}^{\lfloor(N-\lambda) / 2\rfloor}\left(\begin{array}{l}
N \\
k
\end{array}\right)
$$

which clearly depends on the SW length $N$ and on the predetermined threshold $\lambda$.

The probability of missed detection can be evaluated starting from the probability that more than a given number of errors occur in $N$ symbols, given $\mathcal{H}_{1}$. If $p$ is the BSC error probability, we simply have

$$
P_{\mathrm{MD}}=\sum_{k=\lfloor(N-\lambda) / 2\rfloor+1}^{N}\left(\begin{array}{l}
N \\
k
\end{array}\right) p^{k}(1-p)^{N-k} .
$$

We observe that $P_{\mathrm{MD}}$ depends on channel conditions, whereas $P_{\mathrm{EM}}$ is not influenced by the channel. In fact, if SWs are more damaged by channel errors, their detection is harder. Lowering the threshold considered can help the detection, of course, at the expense of an increased probability of emulation.

\section{B. Synchronizing With Soft Values Using the Correlation}

We assume here that soft values are provided to the synchronizer and that the synchronization algorithm is still based on the comparison of the (soft) correlation $\Gamma(\mathbf{r})=\sum_{i=1}^{N} c_{i} r_{i}$ with a threshold $\lambda$

$$
\Gamma(\mathbf{r})=\sum_{i=1}^{N} c_{i} r_{i} \underset{\mathcal{D}_{0}}{\stackrel{\mathcal{D}_{1}}{\gtrless}} \lambda .
$$

Although this choice could appear to be reasonable, there is no theoretical justification for its use when soft values are available and for its superiority with respect to the test based on hard values (43). Indeed, in some cases, the soft-correlation-based test is worse than that based on hard correlation.

For performance evaluation, we start by writing the correlation given $\mathcal{H}_{0}$ as

$$
\Gamma(\mathbf{r})=\sum_{i=1}^{N} d_{i} c_{i}+\sum_{i=1}^{N} n_{i} c_{i} .
$$

This can be seen as the sum of two r.v.s. The first one is given by the sum of $N$ binary r.v.s, while the second is a Gaussian r.v. given by the sum of $N$ Gaussian r.v.s.

After some algebra, the pdf of the soft correlation given $\mathcal{H}_{0}$ is [23]

$$
f_{\Gamma}(\gamma)=\left(\frac{1}{2}\right)^{N} \sum_{k=0}^{N}\left(\begin{array}{l}
N \\
k
\end{array}\right) \frac{1}{\sqrt{2 \pi N} \sigma} e^{-(\gamma-N+2 k)^{2} / 2 N \sigma^{2}} .
$$

It should be noted that, for low-noise standard deviation values, the sum of the bell-shaped curves maintains the individual curve shape, whereas the sum smooths the total curve for higher standard deviation values. 


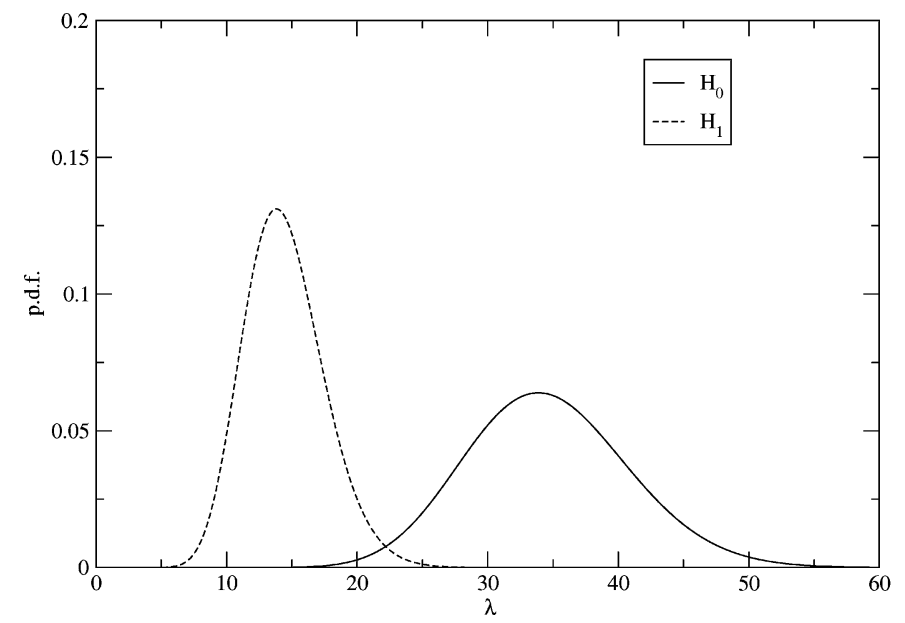

(a)

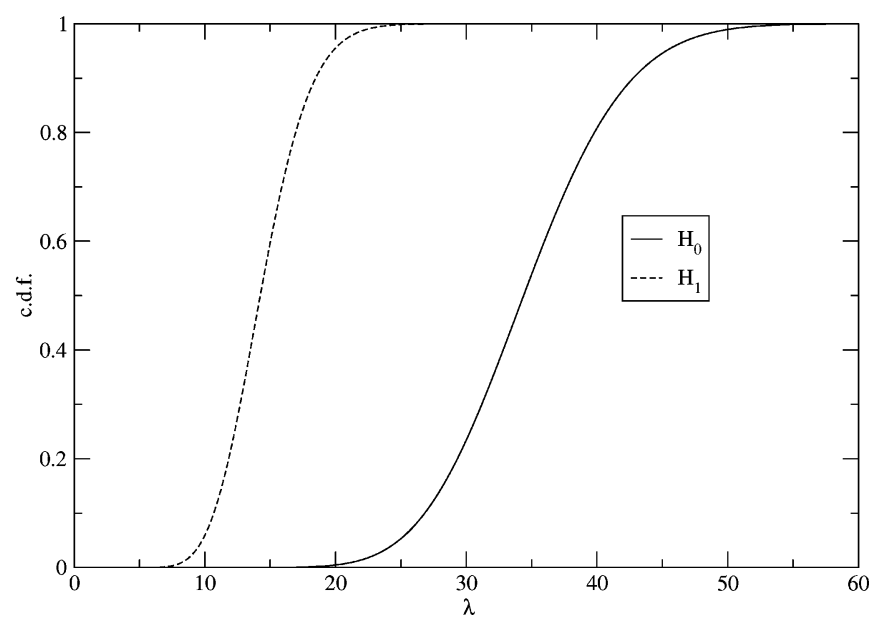

(b)

Fig. 3. (a) pdf and (b) cdf of the LLR metric under hypotheses $\mathcal{H}_{0}$ and $\mathcal{H}_{1}$. SW length $N=32, E_{s} / N_{0}=1 /\left(2 \sigma^{2}\right)=-5 \mathrm{~dB}$.

In order to evaluate the probability of emulation, we need to integrate the pdf in (50) from the threshold $\lambda$ to infinity, obtaining

$$
P_{\mathrm{EM}}=\left(\frac{1}{2}\right)^{N+1} \sum_{k=0}^{N}\left(\begin{array}{l}
N \\
k
\end{array}\right) \operatorname{erfc}\left(\frac{\lambda-N+2 k}{\sqrt{2 N} \sigma}\right) .
$$

On the other hand, the correlation given $\mathcal{H}_{1}$ can be written as

$$
\Gamma(\mathbf{r})=\sum_{i=1}^{N} c_{i}^{2}+\sum_{i=1}^{N} n_{i} c_{i}=N+\sum_{i=1}^{N} n_{i} c_{i} .
$$

It is thus

$$
\operatorname{Pr}\left\{\Gamma(\mathbf{R})<\lambda \mid \mathcal{H}_{0}\right\}=\operatorname{Pr}\left\{\sum_{i=1}^{N} n_{i} c_{i}<\lambda-N\right\} .
$$

The summation term is a Gaussian r.v., being the sum of $N$ independent Gaussian r.v.s, each with zero mean and variance $\sigma^{2}$. It is thus easy to evaluate the probability of missed detection as

$$
P_{\mathrm{MD}}=\frac{1}{2} \operatorname{erfc}\left(\frac{N-\lambda}{\sqrt{2 N} \sigma}\right)
$$

It is worthwhile to remark that, regardless of the common engineering practice of preferring soft correlation, synchronizers based on hard correlation can provide better results for some channel conditions.

\section{NUMERICAL RESULTS}

We report in this section some examples of numerical results obtained with the analysis above. In particular, we compare the LRT with its generalized version and with correlation-based tests. The analysis in all of the cases shown in the following has been validated through simulation; a perfect match has been observed, as expected, since the analysis is exact.

First, we report in Fig. 3 the pdf and cdf, respectively, of the LLR under hypothesis $\mathcal{H}_{0}$. In these figures, the $\mathrm{SW}$ is composed of $N=32$ bits, and the noise is characterized by a value of
$E_{s} / N_{0}=1 /\left(2 \sigma^{2}\right)=-5 \mathrm{~dB}$. These curves have been evaluated by using (27). Note that the probability of emulation or false alarm with a given threshold $\lambda$ is exactly the cdf in Fig. 3 for $\mathcal{H}_{0}$. In the same figures, the distributions under hypothesis $\mathcal{H}_{1}$ are also shown. In this case, the cdf in Fig. 3 for $\mathcal{H}_{1}$ gives the missed detection probability for a given threshold $\lambda$.

As an example of application of the presented analysis for GLRT, we report in Fig. 4 the distributions of the metric under hypothesis $\mathcal{H}_{0}$, for the probability of emulation, and under hypothesis $\mathcal{H}_{1}$, for the probability of correct detection $P_{D}=1-$ $P_{\mathrm{MD}}$. These figures have been obtained from the ch.f. (37) with (40) and (42), for the same conditions as Fig. 3.

In the comparison, LRT and GLRT perform almost the same, except under very bad channel conditions. We will report consequently the comparison results for very low SNRs, where the performance of the two metrics presents a visible discrepancy.

In this regard, in order to compare the proposed LRT metric with the GLRT and the correlation metrics (both with hard and soft decisions), we show in Fig. 5 the ROC curves, for the case $N=32$ and $E_{s} / N_{0}=-5 \mathrm{~dB}$. The probabilities of emulation (false alarm) $P_{\mathrm{EM}}$ and of correct detection $P_{\mathrm{D}}$ are represented in the axes, parameterized by the threshold values. The corresponding curves obtained with the correlation metric are reported for comparison. We can observe that a high gain can be achieved with respect to correlation with the derived LRT metric, for any value of $P_{\mathrm{D}}$, and that GLRT suffers only a slight performance degradation.

A similar behavior is confirmed for $E_{s} / N_{0}=-8 \mathrm{~dB}$ in Fig. 6, where again it can be observed that the optimum metric outperforms the correlation metrics as expected. In this case of very bad channel conditions, the performance gap between the LRT and GLRT metrics is more evident. Moreover, note that for this extreme channel condition, GLRT and soft correlation perform similarly.

In Fig. 7, we show the ROC curves for an SW of $N=24$ bits and $E_{s} / N_{0}=0 \mathrm{~dB}$. Moreover, we report in the same figure the results for the "mixed data" case, where the test is applied to a vector composed of random data in the first (last) $j$ positions, followed (preceded) by the shifted versions of the SW for the 


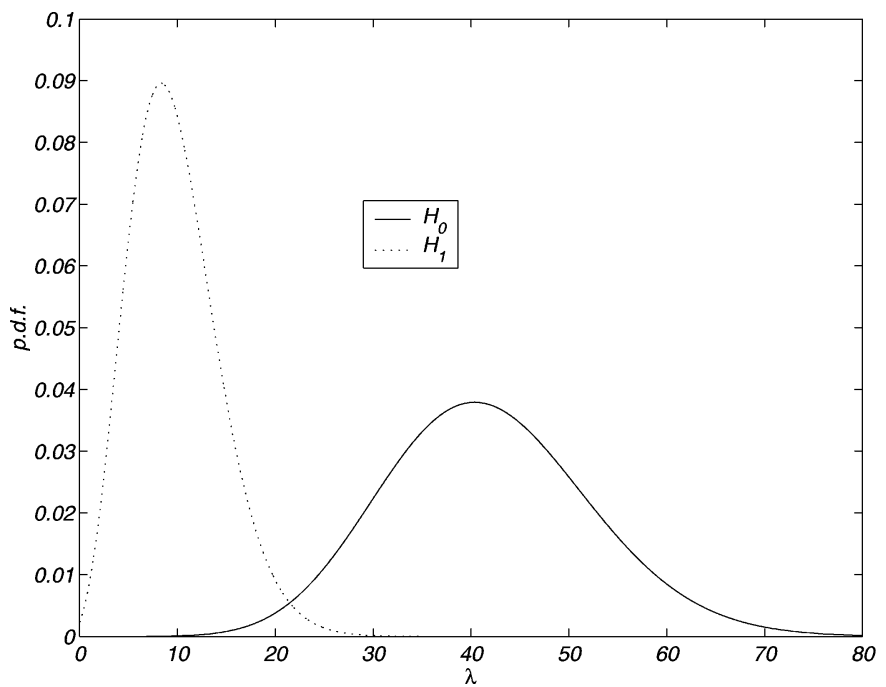

(a)

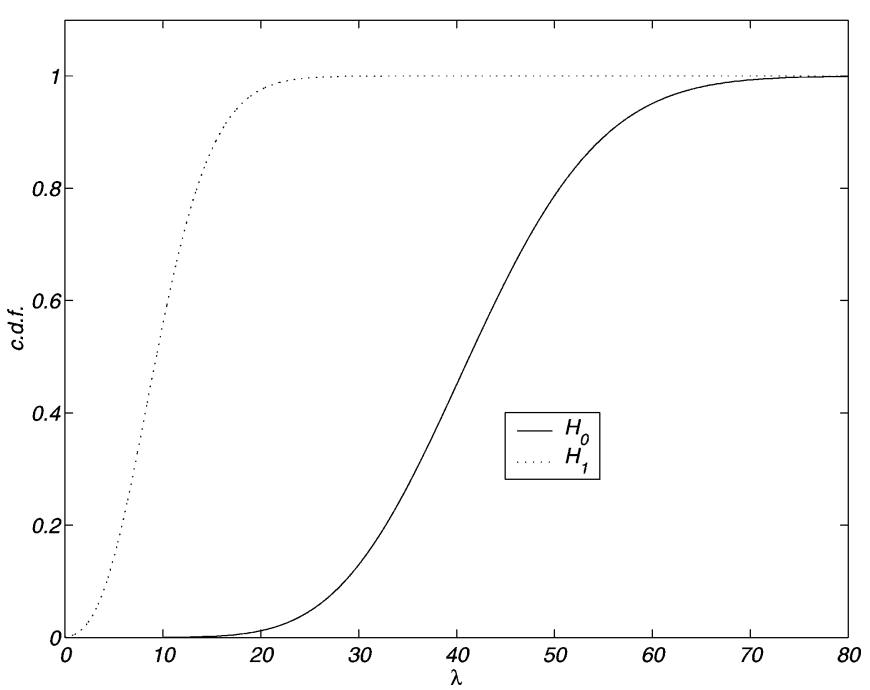

(b)

Fig. 4. (a) pdf and (b) cdf of the GLRT metric under hypotheses $\mathcal{H}_{0}, \mathcal{H}_{1}$. SW length $N=32$ bits, $E_{s} / N_{0}=1 /\left(2 \sigma^{2}\right)=-5$ dB.

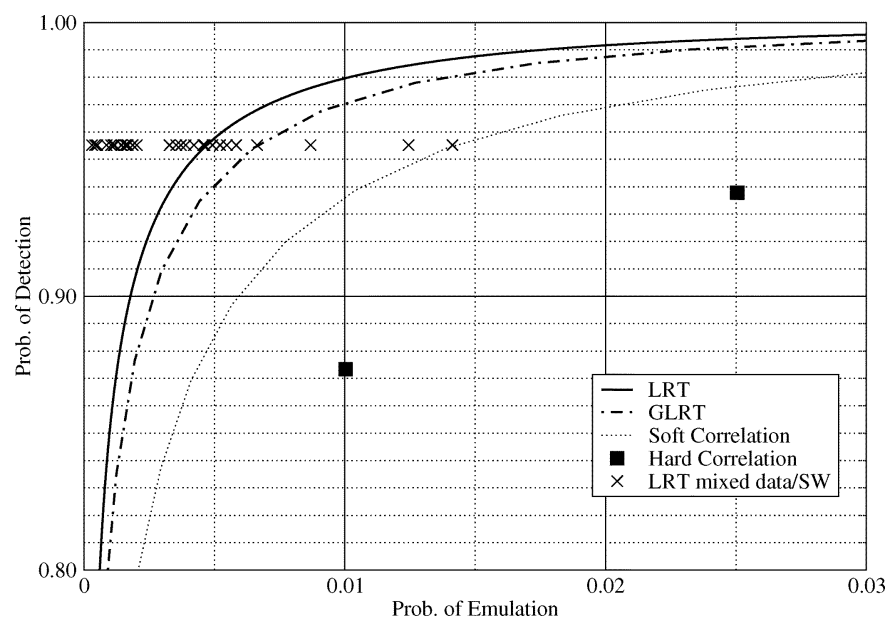

Fig. 5. ROC curves. SW length $N=32$ bits, $E_{s} / N_{0}=1 /\left(2 \sigma^{2}\right)=-5 \mathrm{~dB}$.

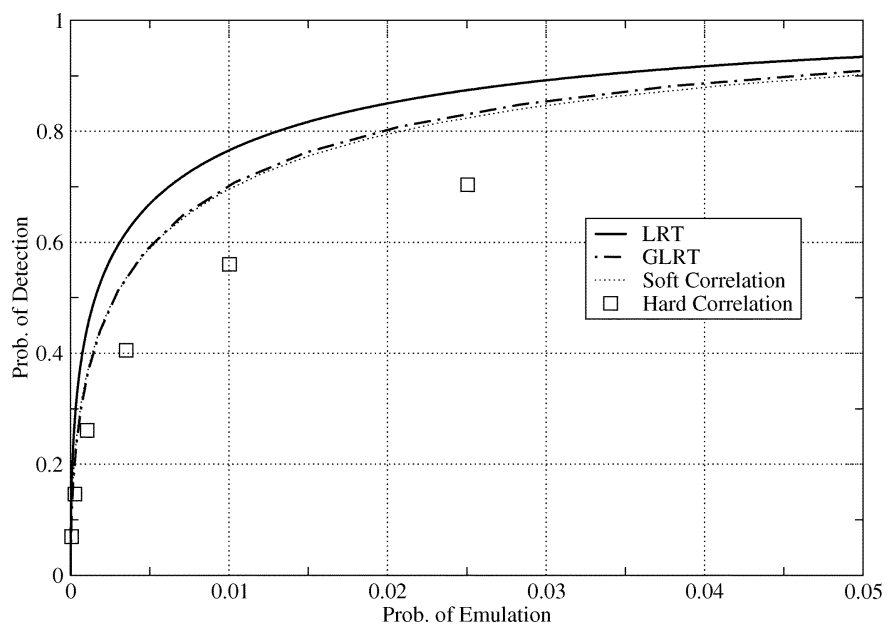

Fig. 6. ROC curves. SW length $N=32$ bits, $E_{s} / N_{0}=1 /\left(2 \sigma^{2}\right)=-8 \mathrm{~dB}$.

remaining $N-j$ positions, with $j=1, \ldots, N-1$. The analysis of the probability of emulation in this case can be easily obtained as in Section IV-A, by the ch.f. derived starting from (22) for the samples in the data part, and from (29) with $c_{i} c_{i-j}$,

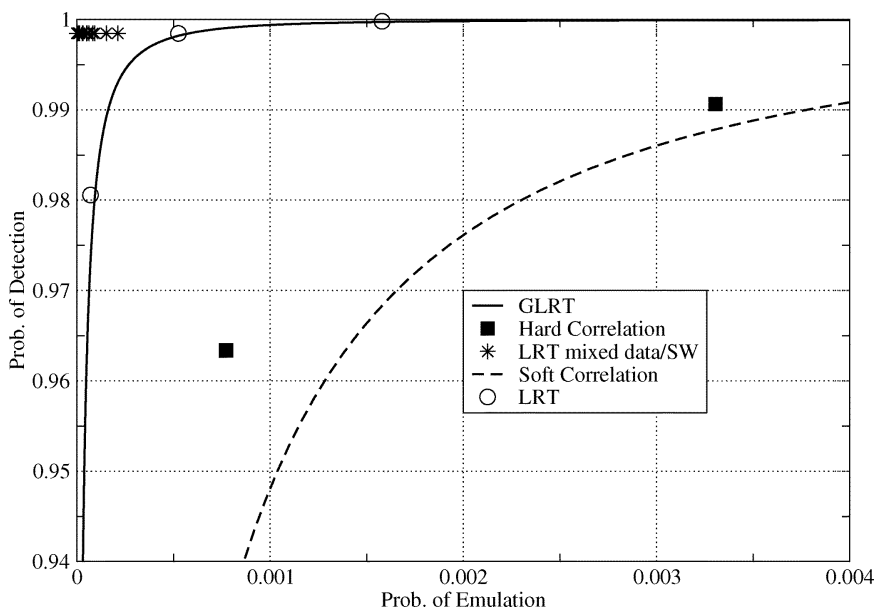

Fig. 7. ROC curves. SW length $N=24$ bits, $E_{s} / N_{0}=1 /\left(2 \sigma^{2}\right)=0 \mathrm{~dB}$.

$i=j+1, \ldots, N$, in place of $c_{i}^{2}$ for the samples due to the portion of the SW. An SW with pattern (in octal) 76571440 [19] is considered in the figure for the "mixed data" case. The asterisks in the top left corner represent the couples $\left(P_{\mathrm{EM}}, P_{\mathrm{MD}}\right)$ in the "mixed data" case for the $N-1=23$ different values of $j$, where a threshold $\lambda=15$ is assumed in (12). We may observe that, as expected, the probability of missed detection does not change with the position $j$, whereas the probability of emulation varies with the position $j$ considered. These simulation points show that here "mixed data" is always (for all $j$ 's) better than pure random data. More generally, we observed that the behavior of the test in the "mixed data" case depends on the specific SW chosen and on channel conditions. For instance, we also report in Fig. 5 the couples $\left(P_{\mathrm{EM}}, P_{\mathrm{MD}}\right)$ obtained in the mixed data case, with a threshold $\lambda=20$ for the SW (in octal) 37562164456 [24]. In this case for most, but not all, values of $j$, the $P_{\mathrm{EM}}$ is below the random data case.

Finally, in order to show a more comprehensive comparison among the different tests, we evaluated the probability of detection versus SNR. The curves for false $\mathrm{SW}$ detection probabilities $P_{\mathrm{EM}}=10^{-4}, P_{\mathrm{EM}}=10^{-2}$ and $P_{\mathrm{EM}}=10^{-5}, P_{\mathrm{EM}}=10^{-3}$ 


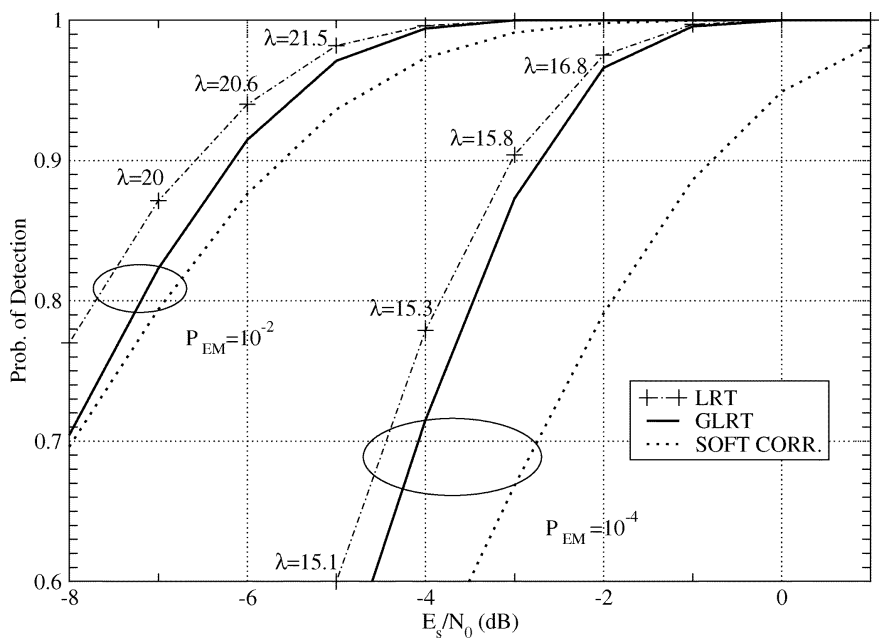

Fig. 8. Probability of detection versus SNR for false SW detection probability $P_{\mathrm{EM}}=10^{-4}$ and $P_{\mathrm{EM}}=10^{-2}$. SW length $N=32$ bits.

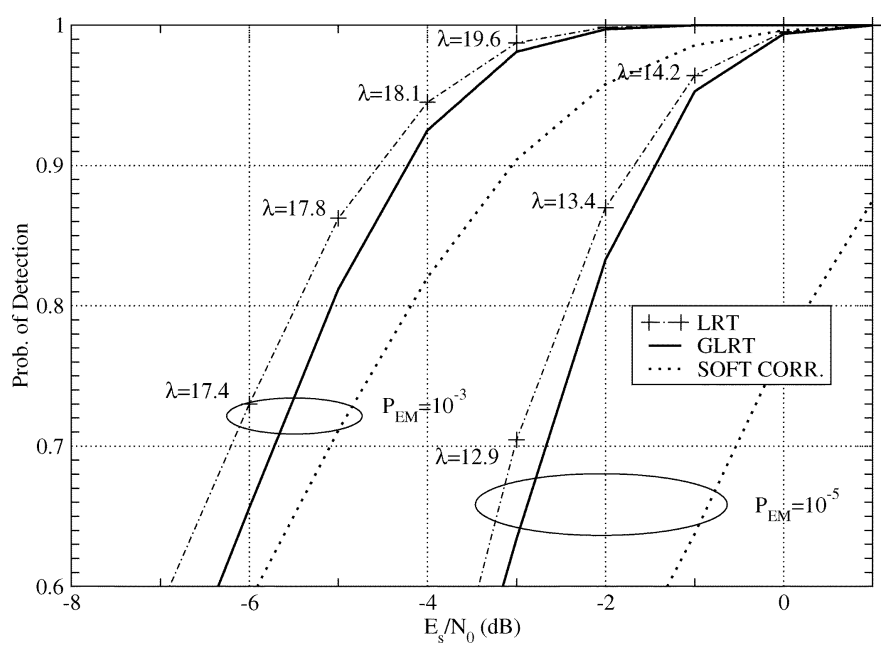

Fig. 9. Probability of detection versus SNR for false SW detection probability $P_{\mathrm{EM}}=10^{-5}, P_{\mathrm{EM}}=10^{-3}$. SW length $N=32$ bits.

are shown in Figs. 8 and 9, respectively. In the LRT curves, the threshold values according to (12) are also reported. It is confirmed that LRT and GLRT greatly outperforms soft correlation. For example, for $P_{\mathrm{EM}}=10^{-4}$ and a target $P_{\mathrm{D}}=0.9$, the synchronizer based on GLRT metric requires, in terms of $E_{s} / N_{0}$, about $2 \mathrm{~dB}$ less than that using soft correlation.

\section{CONCLUSION}

Sequential frame synchronization for aperiodically embedded SWs has been afforded in this paper. According to the hypothesis testing approach and the LRT, along with the GLRT, the relevant detection metrics have been derived.

An analytical performance evaluation of the synchronizers based on LRT and GLRT metrics has been given and represented in terms of ROC curves for antipodal signaling and uniformly distributed data symbols.

In order to have a benchmark for the new metric, the performance of the correlation metrics has also been considered and represented in terms of ROC curves.
A high gain is obtained with both the derived metrics with respect to commonly used correlation-based frame synchronizers.

Since the GLRT metric is simpler to implement and loses very little in performance with respect to optimal LRT for practical SNRs, GLRT-based synchronizers could be preferred in most cases. For very low SNRs, the gap between the two becomes more evident, and resorting to LRT may give substantial performance improvements.

\section{REFERENCES}

[1] R. H. Barker, "Group synchronization of binary digital systems," in Communication Theory. London, U.K.: W. Jackson, 1953.

[2] J. J. Stiffler, Theory of Synchronous Communications. Englewood Cliffs, NJ: Prentice-Hall, 1971.

[3] R. A. Scholtz, "Frame synchronization techniques," IEEE Trans. Commun., vol. COM-28, no. 8, pp. 1204-1213, Aug. 1980.

[4] C. Georghiades and E. Serpedin, Communication Handbook. Boca Raton, FL: CRC, 2002, ch. 18.

[5] R. Talluri, "Error-resilient video coding in the ISO MPEG-4 standard," IEEE Commun. Mag., vol. 36, no. 6, pp. 112-119, Jun. 1998.

[6] Y. Wang, S. Wenger, J. Wen, and A. K. Katsaggelos, "Error-resilient video coding techniques," IEEE Signal Process. Mag., vol. 17, no. 4, pp. 61-82, Apr. 2000.

[7] J. L. Massey, "Optimum frame synchronization," IEEE Trans. Commun., vol. COM-20, no. 4, pp. 115-119, Apr. 1972.

[8] S. W. Golomb and R. A. Scholtz, "Generalized Barker sequences," IEEE Trans. Inf. Theory, vol. IT-11, no. 4, pp. 533-537, Oct. 1965.

[9] S. W. Golomb and M. Z. Win, "Recent results on polyphase sequences," IEEE Trans. Inf. Theory, vol. 44, no. 2, pp. 817-824, Mar. 1998.

[10] G. L. Lui and H. H. Tan, "Frame synchronization for Gaussian channels," IEEE Trans. Commun., vol. COM-35, no. 8, pp. 818-829, Aug. 1987.

[11] P. T. Nielsen, "Some optimum and suboptimum frame synchronizers for binary data in Gaussian noise," IEEE Trans. Commun., vol. COM-21, no. 6, pp. 770-772, Jun. 1973.

[12] P. F. Driessen, "Performance of frame synchronization in packet transmission using bit erasure information," IEEE Trans. Commun., vol. 39, no. 4, pp. 567-573, Apr. 1991.

[13] M. M. K. Howlader and B. D. Woerner, "Decoder-assisted frame synchronization for packet transmission," IEEE J. Sel. Areas Commun., vol. 19, no. 12, pp. 2331-2345, Dec. 2001.

[14] P. T. Nielsen, "On the expected duration of a search for a fixed pattern in random data," IEEE Trans. Inf. Theory, vol. IT-19, no. 9, pp. 702-704, Sep. 1973.

[15] A. Kopansky and M. Bystrom, "Detection of aperiodically embedded syncronization patterns," IEEE Trans. Wireless Commun., vol. 3, no. 5, pp. 1386-1392, Oct. 2004.

[16] C. W. Helstrom, Statistical Theory of Signal Detection. New York: Pergamon, 1978

[17] H. L. Van Trees, Detection, Estimation and Modulation Theory: Part I. New York: Wiley, 1968.

[18] J. L. Maury and F. Styles, "Development of optimum frame synchronization codes for Goddard Space Flight Center PCM telemetry standards," in Proc. Nat. Telemetering Conf., Los Angeles, CA, Jun. 1964, pp. 1-10.

[19] F. Neuman and L. Hofman, "New pulse sequences with desirable correlation properties," in Proc. Nat. Telemetry Conf., Washington, DC, Apr. 1971, pp. 272-282.

[20] M. Chiani and M. G. Martini, "Practical frame synchronization for data with unknown distribution on AWGN channels," IEEE Commun. Lett., vol. 9, no. 5, pp. 456-458, May 2005.

[21] M. Chiani, D. Dardari, and M. K. Simon, "New exponential bounds and approximations for the computation of error probability in fading channels," IEEE Trans. Wireless Commun., vol. 2, no. 4, pp. 840-845, Jul. 2003.

[22] M. Abramowitz and I. A. Stegun, Handbook of Mathematical Functions with Formulas, Graphs, and Mathematical Tables. Washington, DC: U.S. Dept. Commerce, 1970.

[23] M. Chiani and M. G. Martini, "Optimum synchronization of frames with unknown, variable lengths on Gaussian channels," in Proc. IEEE Global Telecomm. Conf., Dallas, TX, Nov. 2004, pp. 4087-4091.

[24] R. Turyn, "Sequences with small correlation," in Error Correcting Codes, H. B. Mann, Ed. New York: Wiley, 1968. 


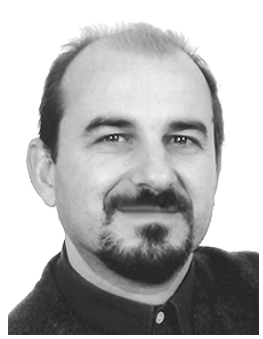

Marco Chiani (M'94-SM'02) was born in Rimini, Italy, in April 1964. He received the Dr. Ing. degree (magna cum laude) in electronic engineering and the $\mathrm{Ph} . \mathrm{D}$. degree in electronic and computer science from the University of Bologna, Bologna, Italy, in 1989 and 1993, respectively.

During the summer of 2001, he was a Visiting Researcher with AT\&T Research, Middletown, NJ. Currently, he is a Research Affiliate with the Massachusetts Institute of Technology, Cambridge. He is also a Full Professor and Chair in Telecommunications with the II Engineering Faculty, University of Bologna. His research interests include wireless communication systems, MIMO systems, wireless multimedia, lowdensity parity-check codes (LDPCC), and ultra-wideband systems. In the framework of the European research program PROMETHEUS, he worked on shortrange millimeter-wave communication systems for Advanced Road Transport Telematics. He is leading the research unit of CNIT/University of Bologna on Joint Source and Channel Coding for wireless video (IST FP6, project Phoenix). $\mathrm{He}$ is a consultant to the European Space Agency (ESA-ESOC) for the design and evaluation of error-correcting codes based on LDPCC for space CCSDS applications.

Prof. Chiani is the former President of the Radio Communications Committee, IEEE Communication Society, and he is the Editor for Wireless Communication for the IEEE TRANSACTIONS ON COMMUNiCATIONS. He chaired and organized sessions at several IEEE International Conferences. He was the Co-Chair of the Wireless Communications Symposium, IEEE ICC 2004, Paris, France.

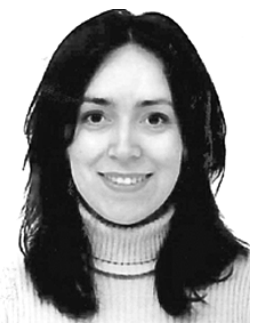

Maria G. Martini (S'99-M'02) received the Laurea degree in electronic engineering (magna cum laude) from the University of Perugia, Perugia, Italy, in 1998 and the Ph.D. degree in electronics and computer science from the University of Bologna, Bologna, Italy, in 2002 .

She was a visiting student with the University of Liège, Liège, Belgium, from 1995 to 1996. After a collaboration with the Aerospace Engineering Department, University of Rome, Rome, Italy, in February 1999 she joined the Dipartimento di Elettronica, Informatica e Sistemistica (DEIS), University of Bologna. Here, she has been a key person for several national and international projects, such as the Joint Source and Channel Coding (JSCC) project, in cooperation with Philips Research Monza and Philips Research France, the Joint Source and Channel Coding Driven Digital Baseband Design for 4G Multimedia Streaming (JOCO), and PHOENIX (Jointly Optimizing Multimedia Transmission in IP Based Wireless Networks) European IST projects. She is currently a Postdoctoral Researcher with CNIT-DEIS, University of Bologna. Her research interests are mainly in video coding, channel coding, joint source and channel coding, error-resilient video transmission, wireless multimedia networks, decision theory, and frame synchronization. She is the author of several patents in the field of wireless video transmission. 